\title{
ANÁLISE HISTOQUÍMICA DA LÍNGUA DE MORCEGOS FRUGÍVOROS NEOTROPICAIS (MAMMALIA - CHIROPTERA) COM RELATO DE PIGMENTAÇÃO EPITELIAL
}

\section{HISTOCHEMICAL ANALYSIS OF THE TONGUE IN NEOTROPICAL FRUGIVOROUS BATS (MAMMALIA - CHIROPTERA) WITH REPORT OF EPITHELIAL PIGMENTATION}

\author{
Eveline de Cássia Batista de Almeida Alves ${ }^{1^{\star}}$ \\ Maria Juliana Gomes Arandas ${ }^{1}$ \\ Nivaldo Bernardo de Lima-Junior ${ }^{1}$ \\ Joaquim Evêncio Neto ${ }^{1}$ \\ Katharine Raquel Pereira dos Santos ${ }^{2}$ \\ 'Universidade Federal Rural de Pernambuco, Recife, PE, Brasil \\ 2Universidade Federal de Pernambuco, Vitória de Santo Antão, PE, Brasil \\ *Autora para correspondência - evelinecassia27@hotmail.com
}

\section{Resumo}

A língua é considerada um órgão-chave para os morcegos, pois os caracteres especializados são utilizados para fins taxonômicos e sistemáticos, estudos cladísticos e elucidação dos padrões dos hábitos alimentares. Objetivou-se caracterizar morfologicamente a língua das espécies Artibeus lituratus, Artibeus planirostris, Artibeus obscurus e Dermanura cinerea e relatar a primeira evidência de melanina no epitélio lingual de morcegos. Foram utilizados 20 exemplares coletados nos municípios de Vitória de Santo Antão e Sirinhaém, em Pernambuco. As línguas foram extraídas e submetidas a técnicas histológicas de rotina, coradas com hematoxilina e eosina (HE) e FontanaMasson. Nas quatro espécies, foi observado o epitélio estratificado pavimentoso queratinizado, porém com presença de grânulos de melanina na camada basal somente nas espécies $A$. lituratus, A. planirostris e A. obscurus, evidenciados por meio da técnica de coloração por Fontana-Masson. A melanina encontrada na língua dessas espécies caracteriza-se como o primeiro registro para a ordem Chiroptera e pode estar relacionada à assepsia oral e/ou à inibição de substâncias tóxicas presentes em vegetais. Portanto, há necessidade de novos estudos, visando aprimorar o conhecimento acerca da função do pigmento nesse órgão.

Palavras-chave: Artibeus; Dermanura; melanina; morcegos; morfologia lingual.

\begin{abstract}
The tongue is regarded as a key organ for bats, since the specialized characters are used for taxonomic and systematic purposes, cladistic studies, and elucidation of patterns of eating habits. This study aimed to characterize morphologically the tongue in the species Artibeus lituratus, Artibeus planirostris, Artibeus obscurus, and Dermanura cinerea, and to report the first evidence of
\end{abstract}


melanin in the lingual epithelium of bats. We used 20 specimens collected in the municipalities of Vitória de Santo Antão and Sirinhaém, in Pernambuco State, Brazil. The tongues were extracted and submitted to routine histological techniques, stained with hematoxylin and eosin (HE) and Fontana-Masson. In the four species, the keratinized stratified pavement epithelium was observed, but with the presence of melanin granules on the basal layer only in the species A. lituratus, A. planirostris, and A. obscurus, evidenced through the Fontana-Masson staining technique. The melanin found in the tongue of these species is characterized as the first record for the order Chiroptera and it may be related to oral asepsis, and/or inhibition of toxic substances observed in plants. Therefore, there is a need for further studies, aiming to improve our knowledge of the function of pigment in this organ.

Keywords: Artibeus; Dermanura; melanin; bats; lingual morphology.

Recebido em: 13 novembro de 2015

Aceito em 05 dezembro de 2016

\section{Introdução}

Os gêneros Artibeus (Leach, 1821) e Dermanura (Gervais, 1856) estão incluídos na subfamília Stenodermatinae e família Phyllostomidae, amplamente distribuída na América do Sul ${ }^{(1,2)}$. Assim, Artibeus, Koopmania e Dermanura eram classificados como subgêneros de Artibeus, mas a sistemática destes táxons sempre apresentou controvérsias e, muitas vezes, dados imprecisos com relação à sua organização ${ }^{(1,3)}$. Contudo, tomando como base estudos moleculares, atualmente, Dermanura é considerado um gênero válido ${ }^{(2,4,5)}$. Dessa forma, as espécies Artibeus lituratus, Artibeus planirostris e Artibeus obscurus estão inseridas no Gênero Artibeus e subsequentemente no subgênero Artibeus, que consistem em espécies de médio e grande porte; enquanto que Dermanura cinerea pertence ao gênero Dermanura, que compreende espécies de pequeno porte. Essas espécies apresentam ampla distribuição no Brasil ${ }^{(2,6)}$ e possuem hábito alimentar predominantemente frugívoro; entretanto, podem incluir em sua dieta néctar, folhas e insetos ${ }^{(7)}$, apresentando, assim, uma ampla plasticidade na adaptação a ambientes antropizados ${ }^{(8)}$.

Estudos sobre caracterização morfológica da língua dos quirópteros fornecem subsídios para a compreensão e elucidação de preferências alimentares, padrões de evolução dos hábitos, fins taxonômicos e sistemáticos e entendimento das adaptações e atuais condições ambientais nos respectivos habitats ${ }^{(3,9-12)}$. Por se tratar de um órgão-chave, devido à grande quantidade de estruturas especializadas, pode se distinguir entre as espécies e em função de suas dietas. Gimenez ${ }^{(9)}$ descreveu a morfologia da língua de espécies de morcegos da família Phyllostomidaee e constatou que este órgão possui diferenças morfológicas relacionadas à grande variedade de hábitos alimentares, permitindo estudos cladísticos do grupo. Outros autores também realizaram estudos envolvendo as espécies da família Phyllostomidae, em que verificaram particularidades na morfologia lingual dos quirópteros ${ }^{(3,13-17)}$. Contudo, trabalhos descritivos que utilizam os métodos histológicos ainda são escassos.

Estudos sobre a caracterização morfológica em outras espécies de vertebrados já relataram a presença de melanina no epitélio lingual de girafas ${ }^{(18)}$ e de lagartos ${ }^{(19)}$, bem como em outros órgãos 
do trato digestório, apresentando nestes diferentes funções ${ }^{(19-22)}$. Em algumas espécies de morcegos das famílias Pteropidae, Megadermatidae, Myzopodidae e Vespertilionidae já foi constatada pigmentação na região do escroto, cuja função está relacionada à proteção do tecido germinativo contra as ações e efeitos da radiação ultravioleta ${ }^{(23)}$.

Tendo em vista esses aspectos, o presente estudo objetivou caracterizar a língua de A. lituratus, A. planirostris, A. obscurus e D. cinerea, com a descrição de uma nova característica para espécies de morcegos Neotropicais.

\section{Material e Métodos}

Foram utilizados 20 espécimes de morcegos, sendo cinco de cada espécie (A. lituratus, A. planirostris, A. obscurus e D. cinerea), coletados através de redes de neblina, em duas localidades: na área urbana do Município de Vitória de Santo Antão e em fragmentos de Mata Atlântica situados na cidade de Sirinhaém, estado de Pernambuco. As coletas foram devidamente autorizadas pelo ICMBio - Instituto Chico Mendes de Conservação da Biodiversidade, através do SISBIO - Sistema de Autorização e Informação em Biodiversidade, com, respectivamente, as seguintes numerações: 16070-1 e 16301-1, bem como foram aprovadas pelo Comitê de Ética CEUA de autorização de $\mathrm{n}^{\circ}$ 23076.049197/2014-19.

Para a obtenção das línguas, os espécimes capturados foram eutanasiados com dose letal de anestésico xilazina $(150 \mathrm{mg} / \mathrm{kg})$ na região peritoneal e, posteriormente, as línguas foram extraídas após a remoção do crânio dos espécimes, previamente fixados com formaldeído tamponado a 10\% por 24 horas. O corte foi realizado à sua base, tomando como referência o orifício epiglótico, para manter a integridade da estrutura.

O material coletado e fixado de acordo com as descrições acima foi clivado longitudinalmente e posteriormente submetido às técnicas histológicas de rotina segundo $\mathrm{Behmer}^{(24)}$, sendo desidratados em álcool etílico em concentrações crescentes até o álcool absoluto, diafanizados em xilol, impregnados e incluídos em parafina. Os blocos obtidos foram cortados em micrótomo ajustado para $5 \mu \mathrm{m}$ e, na sequência, os cortes foram corados pelos corantes Hematoxilina e Eosina (HE) e Fontana Masson.

\section{Resultados e Discussão}

Anatomicamente, as línguas de A. lituratus, A. planirostris e A. obscurus apresentaram coloração grafite em toda a sua extensão. Esse achado está de acordo com o relatado por Gimenez ${ }^{(15)}$. Essa coloração não foi encontrada na espécie $D$. cinerea. 
Histologicamente, as línguas das espécies apresentaram um epitélio estratificado pavimentoso não queratinizado na superfície ventral. Já na região dorsal, observou-se o epitélio estratificado pavimentoso com uma camada córnea sobre as papilas filiformes, com elevado grau de queratinização na língua de A. lituratus, A. planirostris e A. obscurus. Na camada basal de todo o epitélio lingual dessas três espécies, observou-se a presença de melanina, a qual foi confirmada através da coloração Fontana Masson (Fig. 1B), não sendo observada presença de melanina na espécie D. cinerea (Fig. 1C e 1D). Esse elevado grau de queratinização, encontrado nas papilas filiformes da língua de A. lituratus, A. planirostris e A. obscurus, é semelhante ao descrito na análise realizada com morcegos da espécie Pipistrillus kuhli, originários do Egito e da Síria, que apresentam hábito alimentar, em geral, insetívoro, e nos quais foi possível observar alterações na queratinização do epitélio lingual, quando comparado com outras espécies que possuem dietas distintas, além de diferenças intra-específicas, relacionadas à idade ${ }^{(25)}$.

No estudo realizado por Greenbaum e Phillips ${ }^{(26)}$ com as espécies Leptonycteris sanbornie Leptonycteris nivalis, os autores verificaram também que o epitélio lingual dessas espécies é caracterizado por diversos graus de queratinização. Constatou-se ainda que esse alto nível de queratinização epitelial, presente no epitélio lingual de algumas espécies de morcegos, assemelha-se também à encontrada em línguas de cabras da espécie Capra hircus ${ }^{(27)}$ e em aves da espécie Anser fabalis middendorffii ${ }^{(28)}$, constituindo assim uma película protetora decorrente do atrito com os grãos da ração e gramíneas. Nos morcegos, tal estrutura também forma uma camada no epitélio lingual, capaz de proteger contra a fricção atribuída à ingestão do exoesqueleto quitinoso dos insetos predados por A. planirostris, A. lituratus e A. obscurus, já que estas espécies incluem esse item à sua dieta ${ }^{(29)}$.

A melanina, produzida pelos melanócitos, são células pigmentadas derivadas da crista neural e também de células tronco hematopoiéticas ${ }^{(20)}$. Esse pigmento extracutâneo é encontrado em praticamente todos os organismos vivos e caracteriza-se como uma proteína secretada em forma de grânulos, que funciona como fator de proteção dos queratinócitos localizados na pele, evitando exposição à radiação ultravioleta ${ }^{(30)}$. Todavia, diversos animais que apresentam hábitos noturnos também possuem alto nível de melanina na sua superfície externa, como os morcegos ${ }^{(31)}$.

A melanina foi responsável pela coloração grafite observada na análise macroscópica das espécies e, embora seja o primeiro relato para morcegos, a melanina já foi evidenciada na superfície lingual do lagarto-de-língua-azul, da espécie Scincoides tiliqua, encontrado comumente na Austrália ${ }^{(19)}$. Neste estudo, a função da pigmentação não foi esclarecida. Foi encontrada também na língua de girafas da espécie Giraffa camelo pardalis, atribuindo função semelhante à da pele ${ }^{(18)}$. Outros estudos relataram a presença de melanócitos vicerais em anuros, concluindo-se que os melanomacrófagos residentes no fígado de anfíbios produzem a melanina que atua na defesa contra agentes patológicos ${ }^{(20,21)}$. Há relatos da ocorrência de melanina também nas glândulas anexas do trato digestivo de peixes ${ }^{(22)}$, porém com função desconhecida.

Nos estudos que comparam anatômica e histologicamente a língua de diferentes espécies de morcegos $^{(26,25)}$ não foi descrita a presença de melanina na língua. 

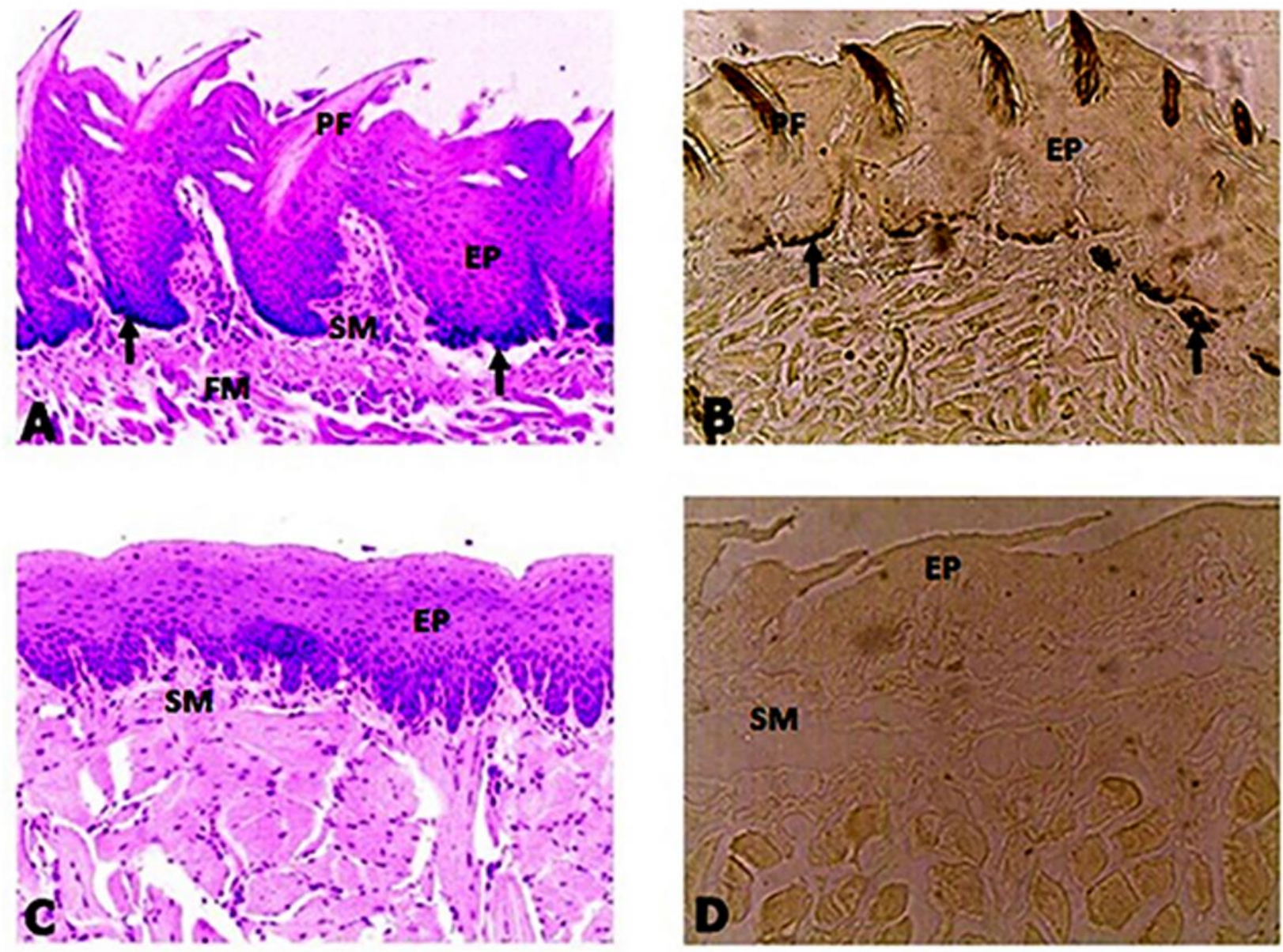

FIGURA 01: Morfologia geral das linguas das espécies estudadas em cortes longitudinais mostrando a superficie dorsal, região médio-posterior. Em A: fotomicrografia da língua de Artibeus lituratus - epitélio estratificado pavimentoso queratinizado (EP), com papilas filiformes (PF); grânulos de melanina (setas), localizados na camada basal (característica evidenciada também em Artibeus planirostris e Artibeus obscurus); logo abaixo, a submucosa (SM); fibras musculares (FM); coloração: hematoxilina e eosina. Em B: fotomicrografia da língua de Artibeus planirostris - observar o epitélio estratificado pavimentoso queratinizado (EP), com papilas filiformes (PF) grânulos de melanina (setas); coloração: Fontana Masson. Em C: fotomicrografia da língua de Dermanura cinerea - o epitélio estratificado pavimentoso queratinizado (EP), submucosa (SM) e ausência de pigmentação; coloração: hematoxilina e eosina. Em D: fotomicrografia da língua de Dermanura cinerea - o epitélio estratificado pavimentoso queratinizado (EP) com ausência de pigmentação; coloração: Fontana Masson. Aumento de 400X.| 


\section{Conclusão}

Foi possível concluir que as características morfológicas evidenciadas nas línguas das espécies estudadas auxiliam no processo inicial de sua digestão e estão estritamente relacionadas às suas dietas. A presença de melanina no epitélio lingual de A. lituratus, A. planirostris e A. obscurus caracterizou-se como o primeiro registro para a ordem Chiroptera e pode estar relacionada à imunologia, promovendo a assepsia oral e/ou a inibição de substâncias tóxicas presentes em vegetais. Portanto, há necessidade de novos estudos, tendo em vista aprimorar o conhecimento acerca da função do pigmento nesse órgão.

\section{Agradecimentos}

À CAPES pela concessão de bolsa de Pós Graduação ao primeiro autor.

\section{Referências}

1. Gardner AL. Order Chiroptera. Mammals of South America (Marsupials, Xenarthras, Shrews, and Bats). Chicago and London: The University of Chicago Press, 2008; 1: 669

2. Reis NR, Peracchi AL, Pedro, WA, Lima IP. Mamíferos do Brasil, 2011, 2: 439.

3. Wetterer AL, Rockman MV, Simmons NB. Phyllostomidae Filogenia de morcegos (Mammalia: Chiroptera): Dados diversos do sistema morfológico, cromossomos sexuais e os locais de restrição. Boletim do Museu Americano de História Natural, 2000, 248:200 pp.

4. Redondo RAF, Brina LPS, Silva RF, Ditchfield AD, Santos FR. Molecular systematics of the genus Artibeus (Chiroptera: Phyllostomidae). Molecular Phylogenetics and Evolution, 2008, 49(1): 44-58.

5. Solari S, Hoofer SR, Larsen PA, Brown AD, Bull RJ, Guerrero JA, Ortega J, Carrera JP, Bradley RD, Baker RJ. Operational criteria for genetically defined species: analysis of the diversification of the small fruit-eating bats, Dermanura (Phyllostomidae: Stenodermatinae). Acta Chiropterologica, 2009, 11(2): 279288.

6. Reis NR, Peracchi AL, Pedro WA, Lima IP. Morcegos do Brasil, Londrina, PR, 2007, 253p.

7. Zortéa M., Mendes SL. Folivory in the big fruit eating bat, Artibeus lituratus (Chiroptera: Phyllostomidae) in eastern Brazil. J. Trop. Ecol., Cambridge, 1993, 9: 117-120.

8. Simmons NB. Ordem Chiroptera. In: WILSON, D. E.; REEDER, D. M. Mammal Species of the World: a taxonomic and geographic reference. Baltimore: Johns Hopkins University Press, 2005, 312-529.

9. Gimenez EA \& Ferrarezzi H. Systematic patterns and evolution of feeding habits in Chiroptera (Archonta: Mammalia). J. Comp. Biol., 1996, 1(3/4): 75-94.

10. Iwasaki S. Evolution of the structure and function of vertebrate tongue. J. Anat. 2002, 201(1):1-13.

11. Abayomi TA, Ofusori DA, Ayoka OA, Odukoya SA, Omotoso EO, Amegor FO, Ajayi AA, Ojo GB, 
Oluwayinka OPA.. Comparative histological study of the tongue of rat (Rattus norvegicus), bat (Eidolon helvum) and pangolin (Manis tricuspis). Int. J. Morphol., 2009, 27(4):1111-1119.

12. Emura S, Okumura T, Chen H. Morphology of the lingual papillae in the Egyptian rousette bat (Rousettus aegyptiacus). Okajimas Folia Anat. Jpn., 2012, 89 (3): 61-66.

13. Mollerach, MI, Mangione S.. Adaptaciones Morfológicas de La lengua de Desmodus rotundus rotundos (Chiroptera: Phyllostomidae) em función de La alimentacion. Mastozool. Neotrop., 2004, 11(2):203-209.

14. Ferrarezzi H, Gimenez EA, Taddei VA. Lingual morphology and cladistic analysis of the New World nectar-feeding bats (Chiroptera: Phyllostomidae). J. Comp. Biol., Ribeirão Preto, 1996, 1(1-2):41-63.

15. Gimenez EA. Dissertação de Mestrado: Morfologia Lingual comparada, filogenia e evolução dos Hábitos alimentares na superfamília Phyllostomidae (Mammalia: Chiroptera). Butucatu/SP, 1993.

16. Arita TH \& Martínez DR. Interacciones flor-murciélago, um enfoque zoocêntrico. Public. Esp. do Instituto de Biologia, 1990, 4: 1-35.

17. Uieda W. Aspectos da morfologia lingual de três espécies de morcegos hematófagos Chiroptera, Phyllostomidae). Rio de Janeiro, Rev. Bras. Biol. 1986, 46(3): 581:587.

18. Feldhamer GA, Drickamer LC, Vassey SH, Merrit J F, Krajewky C. Mammalogy: Adatation, diverty, ecology. Johns Hopkins University Press, Baltimore, 2007, 3: 592 p.

19. Abbate F, Latella G, Montalbano G, Guerrera M.C, Germana GP, Levanti MB. A superfície lingual dorsal do Skink Blue-Tongue (Scincoides tiliqua). Anat Histol, Embryol, 2009, 38: 348-350.

20. Sichel G, Scalia M, Mondio F, Corsaro C. The amphibian Kupffer cells build and demolish melanosomes: an ultrastructural point of view. Pigment Cell Research, 1997, 10: 271-287.

21. Zuasti A, Jiménez-Cervantes C, Garcia-Berrón JC, Ferrer C. The melanogenic system of Xenopus laevis. Archives of Histology and Cytology, 1998, 61:305-316.

22. Lemmes AS, Braccini MC. Descrição e analise histológica das glândulas anexas do trato digestivo de Hoplias malabaricus (Bloch, 1794), (TELEOSTEI, ERYTHRINIDAE). Biodiversidade Pampeana, Uruguaiana, 2004, 2:33-41.

23. KermottLH, Timm RM.. Scrotal Melanins in bats (Chiroptera): Description, distribution and function. J. Zool., 1988, 214: 519-532.

24. Behmer OA, Tolosa EMC, Neto A GF. Manual de Técnicas para Histologia Normal e Patológica, 1976, $1^{a}$ ed. São Paulo: EDART - Ed. da Universidade de São Paulo.

25. Selim A, Nahla NE, Shelfeh M. Comparative Anatomical and Histological Studies of the Tongue between the Egyptian Bat Pipistrillus kuhli and the Syrian Bat Pipstrillus kuhli. Tishreen University Journal for Research and Scientific Studies - Biological Sciences Series, 2008, 30(1): 247-255.

26. Greenbaum IF, Phillips CJ. Comparative Anatomy and General Histology of Tongues of Long-Nosed Bats (Leptonycteris sanborni and L. nivalis) with Reference to Infestation of Oral Mites. Journal of Mammalogy, 1974, 55( 3): 489-504.

27. Fonseca ET, Oliveira CM, Franciolli AL, Miglino MA. Características das papilas o dorso da língua de cabras (Capra hircus): estudo por de microscopia eletrônica de varredura e luz. Pesq. Vet. Bras. 2011, 31(1):67-73.

28. Iwasaki S, Asami T \& Chiba A. Ultrastructural study of the keratinization of the dorsal epithelium of the tongue of Middendorff's Bean goose, Anserfabalis middendorffii (Anseres, Antidae). Anat. Rec. 1997, 247:149-163. 
29. Novaes RLM. \& Nobre CC. Dieta de Artibeus lituratus (Olfers, 1818) em área urbana na cidade do Rio de Janeiro: frugivoria e novo registro de folivoria.Chiroptera Neotropical, 2009, 15(2).

30. Prota G. Recent Advances in the Chemistry of Melanogenesis in Mammals. The Journal of Investigative Dermatology, 1980, 75: 122-127.

31. Burkhart CG, Burkhart CN. The mole theory: primary function of melanocytes and melanin may be antimicrobial defense and immunomodulation (not solarprotection). International Journal of Dermatology, 2005, 44:340-342. 